\title{
Pathological sampling of biliary stenosis: is fluoroscopic guidance sufficient or not?
}

\section{다 (1) $\odot$}

\author{
Author \\ Frederic Prat \\ Institution \\ Endoscopy Unit, Dept of Oncology, Visceral Surgery and \\ Hepatogastroenterology, Cochin Hospital, Paris University, \\ Paris, France \\ Bibliography \\ DOI https://doi.org/10.1055/a-0915-2249 | \\ Endoscopy International Open 2019; 07: E916-E918
}

\author{
(c) Georg Thieme Verlag KG Stuttgart · New York \\ eISSN 2196-9736 \\ Corresponding author \\ Frederic Prat, MD, PhD, Cochin Hospital, Paris University, \\ 27 rue du Faubourg St Jacques, 75014 Paris, France \\ Fax: +33158411965 \\ frederic.prat@aphp.fr
}

Diagnosis of biliary strictures is often straightforward, as in a patient who presents with jaundice, pruritus, a pancreatic head mass on computed tomography (CT) scan and more than $90 \%$ chance of an informative sampling on endoscopic ultrasound-guided fine-needle aspiration (EUS-FNA), or when cholestasis a few months after orthotopic liver transplantation followed by magnetic resonance imaging (MRI) shows an anastomotic stricture, leaving little doubt about the benign nature of the lesion and making cytological sampling even unnecessary. Differentiating between benign and malignant bile duct strictures, however, can be more challenging because strictures involving the bile duct represent a heterogeneous group of benign and malignant conditions with an appearance on $C T, M R$, endosonographic or direct cholangiographic examinations that is non-specific. In a substantial subset of patients, a multidisciplinary approach including clinical examination, biochemical testing such as serum CA19-9, radiographic imaging, endoscopic procedures, and pathological evaluation with ancillary techniques is therefore necessary. During the last three decades, fluoroscopy-guided sampling, in the form of ERCP-guided brush cytology or biopsies has been the mainstay of this diagnostic approach. The sensitivity of detecting malignancy by using these methods, however, ranges between $8 \%$ and $67 \%$ $[1,2]$. To improve detection of malignant strictures, adjunctive techniques were developed that include digital image analysis, KRAS mutational testing and fluorescence in situ hybridization (FISH). However, the sensitivities of these assays remain moderate at best and they have not significantly altered the pattern of indeterminate biliary strictures.

The consequences of this relatively poor diagnostic performance are two-fold: on one hand, the need to obtain a positive diagnosis of malignancy before undergoing surgery leads to additional endoscopic retrograde cholangiopancreatographies
(ERCPs) for diagnostic purposes, with patients whose cytology is negative nearly twice as likely to undergo additional tests than those whose cytology is positive [1]. As a result, therapeutic decisions for malignant strictures are often delayed by several weeks or months, which places patients at risk for disease progression. On the other hand, relying too much on the clinical impression and dismissing negative cytology can result in unnecessary surgical resection. It has been reported that up to $15 \%$ of patients who undergo surgery for a suspected malignant stricture are operated on needlessly for benign disease [3].

To more clearly discern avenues for improvement aimed at reducing the rate of unnecessary surgery as well as an unacceptably lengthy diagnostic workup, we can review what has been explored so far. Notwithstanding other relevant interrogations, there are three points I would like to raise summarily here: What is the best method to collect samples under fluoroscopy; Can the material obtained under fluoroscopy be exploited in a manner that optimizes diagnostic performance, including by using new molecular technologies; and, To what extent can alternative sampling-guiding methods substitute or complement fluoroscopy?

Brushing the stricture with a cytobrush is a standard method and the most commonly used and reported. Because the cytobrush is generally passed over a guidewire, it is easy to apply it along the stricture as it appears on ERCP, but the low yield is generally attributed to the low cellularity of primary cholangiocarcinoma, especially the infiltrating type, with malignant cells embedded in a thick fibrous stroma, which results in brush yielding only isolated cells or small clusters, often so well differentiated that they can be difficult to distinguish from the normal biliary epithelium. New brushes have been designed with longer, thicker, and wiry bristles that can scratch deeper in the neoplastic epithelium and collect more cells of interest; 
a recent study showed that such brushes actually collect more cell clusters, of both large (>50 cells) or medium (>10 cells) size, but failed to show any significant improvement in terms of sensitivity [4]. Another method is to perform biopsies instead of, or as a complement to, brush cytology. In a systematic review and meta-analysis of biliary brush cytology and intraductal biopsy related to nine studies published between 1987 and 2008, Navaneethan et al found the pooled sensitivity and specificity of brushings were $45 \%$ and $99 \%$, respectively, as against $48.1 \%$ and $99.2 \%$, respectively, for biopsies, whereas a combination of both modalities only modestly increased the sensitivity at $59.4 \%$ [2]. One possible reason for this disappointing result is that although transpapillary-guided biopsies can be easily obtained through an endoscopic sphincterotomy, it is nearly impossible to guide the forceps on a selected part of the stricture, with the instrument always capturing tissue at the same site. A very recently launched device (Histoguide) allows for transpapillary-guided biopsy sampling, with a small forceps being guided through a catheter over the wire, but no study has been reported so far of its performance.

Resorting to new technologies to obtain more useful information from the little available material has been relatively disappointing in the past. Digital Image Analysis or testing for KRAS point-mutations has only marginally improved sensitivity, sometimes at the expense of specificity. Only FISH technique, especially in patients with primary sclerosing cholangitis who have dominant strictures, has proven to increase sensitivity without impairing specificity when polysomy is found in more than one subsequent cytobrushing [5]. Perhaps more encouraging is the potential offered by next generation sequencing (NGS) techniques to use all the available material, including cellular debris, to characterize a large range of mutational changes associated with cancer, especially loss of heterozygosity (LOH), as evidenced in a study by Singhi et al: a 28-gene NGS panel ( $\mathrm{Bi}$ liSeq) using ERCP-obtained biliary specimens (including both brushings and biopsies) from 252 patients with bile duct strictures found a high sensitivity and specificity for malignant strictures at $73 \%$ and $100 \%$, respectively. Even more interestingly, the combination of the panel and pathological evaluation increased the sensitivity to $83 \%$ and maintained a specificity of $99 \%$. Specifically, the NGS panel improved the sensitivity of pathological evaluation for malignancy from $35 \%$ to $77 \%$ for biliary brushings and from $52 \%$ to $83 \%$ for biliary biopsies, suggesting an added value for both types of fluoroscopically-guided methods of sampling [6].

Although promising, these methods may not suffice to solve the diagnostic conundrum in patients with truly indeterminate strictures, that is, those beyond the reach of EUS-FNA and with a clinically unpredictable chance of harboring cancer. For those patients, several techniques have been proposed in combination with ERCP and fluoroscopy: intraductal EUS (IDUS) does not add significant information as compared to EUS, ERCP or $M R I / C T$, and is not a reliable method to guide biopsies during ERCP. Probe-based confocal laser endomicroscopy (pCLE), although promising in the late 2000s and early 2010s with a high sensitivity, did not reach a satisfactory specificity and was found unable to clearly differentiate cancer from inflammation.
Over the last 10 years, single-operator cholangioscopy (SOC) has gained popularity and SOC-guided biopsies have been found to be technically feasible along with direct visualization of the stricture, allowing for more accurate targeting of biopsies. In non-selected patients with biliary strictures, SOC has been found in several studies and systematic reviews to exceed $80 \%$ in sensitivity, and to reach a more modest $60 \%$ to $65 \%$ in patients strictly selected as bearing indeterminate strictures as defined above [7]. In addition to SOC's diagnostic value, a soonto-be-published multicenter prospective study from our group [8], found that patient management changed after use of the technique compared to planned management before SOC in $58.3 \%$ of cases, generally in favor of conservative treatment. Although direct cholangioscopic grading has been accused of inaccuracies and poor interobserver agreement, SOC has a potential for further development, including improvement in biopsy sampling with forceps grabbing larger and deeper tissue as well as refinements in visual analysis of the cholangioscopic image, similar to what has been observed in digestive endoscopy with the characterization of Barrett's mucosa or colorectal polyps.

In conclusion, not all biliary strictures require fluoroscopyguided sampling because a majority of patients, provided they are referred to an expert center, can receive an accurate and expeditious diagnosis by means of a thorough analysis of their clinical case and EUS-FNA when applicable. Other challenges remain, and will probably remain in the foreseeable future in the realm of ERCP. Cholangioscopy is already of great help in most difficult cases and its applications almost certainly will be expanded. Meanwhile, the still disappointing performance of brush cytology and fluoroscopy-guided biopsies may be improved if NGS techniques become more affordable and easily accessible.

\section{Competing interests}

Prof. Dr. Prat is a consultant for Boston Scientific and Olympus Europe.

\section{References}

[1] Sethi R, Singh K, Warner B et al. The impact of brush cytology from endoscopic retrograde cholangiopancreatography (ERCP) on patient management at a UK teaching hospital. Frontline Gastroenterol 2016; 7: $97-101$

[2] Navaneethan U, Njei B, Lourdusamy V et al. Comparative effectiveness of biliary brush cytology and intraductal biopsy for detection of malignant biliary strictures: a systematic review and meta-analysis. Gastrointest Endosc 2015; 81: 168-176

[3] Wakai T, Shirai Y, Sakata J et al. Clinicopathological features of benign biliary strictures masquerading as biliary malignancy. Am Surg 2012; 78: $1388-1391$

[4] Bank JS, Witt BL, Taylor L] et al. Diagnostic yield and accuracy of a new cytology brush design compared to standard brush cytology for evaluation of biliary strictures. Diagnost Cytopathol 2018; 46: 234-238 
[5] Modha K, Navaneethan U. Diagnosis and management of primary sclerosing cholangitis perspectives from a therapeutic endoscopist. World J Hepatol 2015; 18: 799-805

[6] Singhi AD, Nikiforova M, Chennat J et al. Integrating next-generation sequencing to endoscopic retrograde cholangiopancreatography (ERCP)-obtained biliary specimens improves the detection and management of patients with malignant bile duct strictures. Gut 2019: doi:10.1136/gutjnl-2018-317817
[7] Navaneethan U, Hasan MK, Lourdusamy V et al. Single-operator cholangioscopy and targeted biopsies in the diagnosis of indeterminate biliary strictures: a systematic review. Gastrointest Endosc 2015; 82: $608-614$

[8] Prat F, Leblanc S, Foissac F et al. Impact of peroral cholangiopancreatoscopy on the management of indeterminate biliopancreatic conditions: a multicenter prospective trial. Frontline Gastroenterol 2018: doi:10.1136/flgastro-2018-100985 